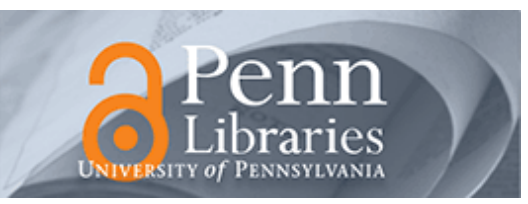

University of Pennsylvania

ScholarlyCommons

\title{
Adaptive Polarization Contrast Techniques for Through-Wall Microwave Imaging Applications
}

\author{
K M. Yemelyanov \\ Villanova University \\ N Engheta \\ University of Pennsylvania, engheta@ee.upenn.edu
}

A Hoorfar

Villanova University

J A. McVay

Follow this and additional works at: https://repository.upenn.edu/ese_papers

\section{Recommended Citation}

K M. Yemelyanov, N Engheta, A Hoorfar, and J A. McVay, "Adaptive Polarization Contrast Techniques for Through-Wall Microwave Imaging Applications", . May 2009.

Yemelyanov, K. M.; Engheta, N.; Hoorfar, A.; McVay, J. A., "Adaptive Polarization Contrast Techniques for ThroughWall Microwave Imaging Applications," Geoscience and Remote Sensing, IEEE Transactions on , vol.47, no.5, pp.1362-1374, May 2009 URL: http://ieeexplore.ieee.org/stamp/

stamp.jsp?arnumber $=4815956 \&$ isnumber $=4815947$

Copyright 2009 IEEE. Reprinted from IEEE Transactions on Geoscience and Remote Sensing, Volume 47, Issue 5, May 2009, pages 1362-1374.

This material is posted here with permission of the IEEE. Such permission of the IEEE does not in any way imply IEEE endorsement of any of the University of Pennsylvania's products or services. Internal or personal use of this material is permitted. However, permission to reprint/republish this material for advertising or promotional purposes or for creating new collective works for resale or redistribution must be obtained from the IEEE by writing to pubs-permissions@ieee.org. By choosing to view this document, you agree to all provisions of the copyright laws protecting it.

This paper is posted at ScholarlyCommons. https://repository.upenn.edu/ese_papers/478

For more information, please contact repository@pobox.upenn.edu. 


\title{
Adaptive Polarization Contrast Techniques for Through-Wall Microwave Imaging Applications
}

\begin{abstract}
In this paper, we describe and utilize polarization contrast techniques of the adaptive polarization difference imaging algorithm and its transient modification for through-wall microwave imaging (TWMI) applications. Originally developed for optical imaging and sensing of polarization information in nature, this algorithm is modified to serve for target detection purposes in a through-wall environment. The proposed techniques exploit the polarization statistics of the observed scene for the detection and identification of changes within the scene and are not only capable of mitigating and substantially removing the wall effects but also useful in detecting motion, when conventional Doppler techniques are not applicable. Applications of the techniques to several TWMI scenarios including both homogeneous and periodic wall cases are presented.
\end{abstract}

\section{Comments}

Yemelyanov, K. M.; Engheta, N.; Hoorfar, A.; McVay, J. A., "Adaptive Polarization Contrast Techniques for Through-Wall Microwave Imaging Applications," Geoscience and Remote Sensing, IEEE Transactions on , vol.47, no.5, pp.1362-1374, May 2009 URL: http://ieeexplore.ieee.org/stamp/

stamp.jsp?arnumber $=4815956 \&$ isnumber $=4815947$

Copyright 2009 IEEE. Reprinted from IEEE Transactions on Geoscience and Remote Sensing, Volume 47, Issue 5, May 2009, pages 1362-1374.

This material is posted here with permission of the IEEE. Such permission of the IEEE does not in any way imply IEEE endorsement of any of the University of Pennsylvania's products or services. Internal or personal use of this material is permitted. However, permission to reprint/republish this material for advertising or promotional purposes or for creating new collective works for resale or redistribution must be obtained from the IEEE by writing to pubs-permissions@ieee.org. By choosing to view this document, you agree to all provisions of the copyright laws protecting it. 


\title{
Adaptive Polarization Contrast Techniques for Through-Wall Microwave Imaging Applications
}

\author{
Konstantin M. Yemelyanov, Member, IEEE, Nader Engheta, Fellow, IEEE, \\ Ahmad Hoorfar, Senior Member, IEEE, and John A. McVay, Member, IEEE
}

\begin{abstract}
In this paper, we describe and utilize polarization contrast techniques of the adaptive polarization difference imaging algorithm and its transient modification for through-wall microwave imaging (TWMI) applications. Originally developed for optical imaging and sensing of polarization information in nature, this algorithm is modified to serve for target detection purposes in a through-wall environment. The proposed techniques exploit the polarization statistics of the observed scene for the detection and identification of changes within the scene and are not only capable of mitigating and substantially removing the wall effects but also useful in detecting motion, when conventional Doppler techniques are not applicable. Applications of the techniques to several TWMI scenarios including both homogeneous and periodic wall cases are presented.
\end{abstract}

Index Terms-Adaptive polarization difference imaging (APDI), polarization, polarization contrast sensing, target detection, through-wall imaging.

\section{INTRODUCTION}

$\mathbf{T}$ HE IMAGING of objects through walls and obstacles using microwave signals offers various applications in civilian and defense scenarios. Ideally, for a through-wall microwave imaging (TWMI) system, one desires to design, construct, and test a portable setup that would be capable of collecting information about objects behind wall(s), process it, and identify and classify those objects. Owing to various constraints and specifications such as signal penetration through wall materials, image range and azimuth resolutions, and portability of the setup, the system is to operate in a microwave band of about 1-3 GHz. A minimum system bandwidth of about $30 \%$ with respect to the center frequency is essential for providing a

Manuscript received May 19, 2008; revised September 14, 2008 and December 23, 2008. Current version published April 24, 2009. This work was supported by the Defense Advanced Research Projects Agency under Grant MDA972-02-1-0022.

K. M. Yemelyanov is with the Center for Advanced Communications, Department of Electrical and Computer Engineering, Villanova University, Villanova, PA 19085 USA (e-mail: konstantin.yemelyanov@ villanova.edu).

N. Engheta is with the Department of Electrical and Systems Engineering, University of Pennsylvania, Philadelphia, PA 19104 USA (e-mail: engheta@ee. upenn.edu).

A. Hoorfar is with the Antenna Research Laboratory, Center for Advanced Communications, Department of Electrical and Computer Engineering, Villanova University, Villanova, PA 19085 USA (e-mail: ahmad.hoorfar@ villanova.edu).

J. A. McVay was with the Center for Advanced Communications, Department of Electrical and Computer Engineering, Villanova University, Villanova, PA 19085 USA. He is now with Eureka Aerospace, Pasadena, CA 91107 USA (e-mail: mcvay@eurekaaerospace.com).

Color versions of one or more of the figures in this paper are available online at http://ieeexplore.ieee.org.

Digital Object Identifier 10.1109/TGRS.2009.2015569 sufficient resolution. Various polarimetric-based techniques for target detection and discrimination have been suggested in the last two decades (see, e.g., [1]-[13], and the references therein). Performances of several well-known polarization-based target detection algorithms are given in [10].

One of the TWMI setups developed in our research group consists of a dual-polarized low-profile antenna array, which is responsible for collecting scattered electromagnetic fields from the observed scene. The scene of interest can be illuminated by a set of transmitters, typically two to four. The receiving array consists of a horizontal array of eight dual-polarized antenna elements, which, in turn, scans vertically through eight positions in the course of the measurement, and thus, 64 locations are covered. The detailed discussion on the design of the array can be found in [14]. The issues concerning the processing of the collected data include both electromagnetics and signal processing aspects. Advances in signal processing algorithms, such as beamforming techniques and methods for the detection of a target location in a through-wall environment have been extensively reported in the literature (see, e.g., [15]-[17]). In this paper, we discuss some electromagnetic aspects of the target detection problem in TWMI based on the analysis of the polarization characteristics of the observed scene. Methods originally developed and successfully utilized for target detection in the optical domain [21] have been extended into the microwave regime with an emphasis on target detection in the TWMI environment.

The polarization-contrast-based algorithms presented in this paper have been evolved from polarization difference imaging (PDI), a bio-inspired technique originally developed for optical imaging [18]-[20]. The optical PDI algorithm provided significant enhancements in target detection and feature extraction over conventional imaging methods. As a next step in the development of the PDI technique, we introduced the concept of adaptive PDI (APDI) in the optical imaging domain [21]. The idea of an adaptive mechanism in polarization vision was also inspired from natural phenomena (see, e.g., [22]). Using principal component (PC) analysis (PCA) on the statistics of the polarization data from the observation scene using two channels that receive two polarization components, we developed a method to "adaptively" determine the two optimum information channels, with appropriate weighting (i.e., multiplicative) coefficients, that can be formed as linear combinations of the two original polarization signal channels. In addition, we determine the proper orientations of polarization filters that can achieve the optimal target-to-background separation, where the "target" is defined as an area with distinct polarization characteristics, as 


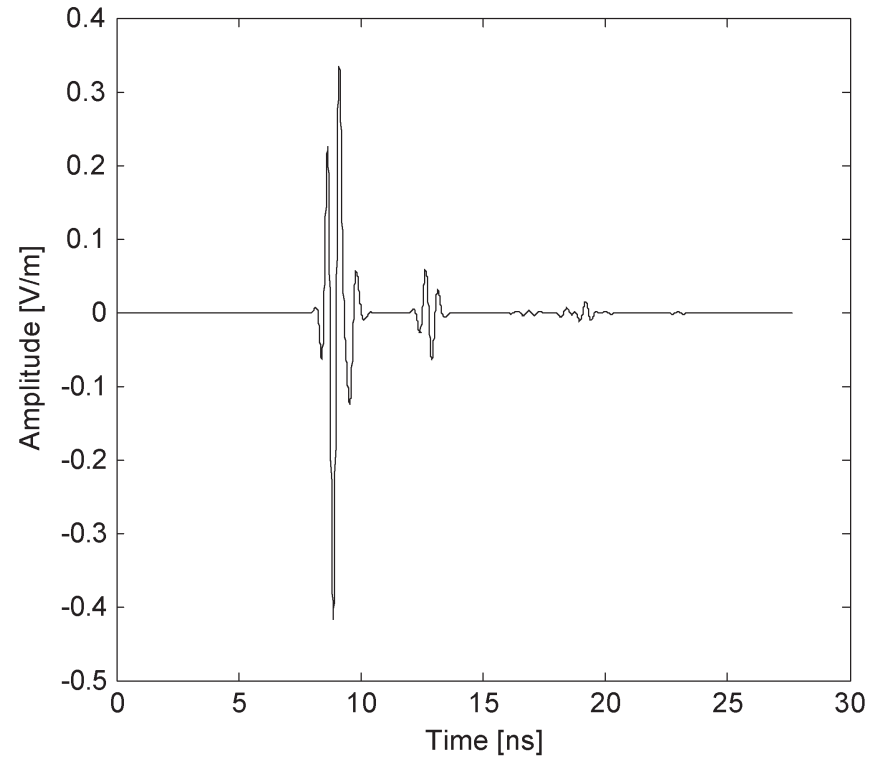

(a)

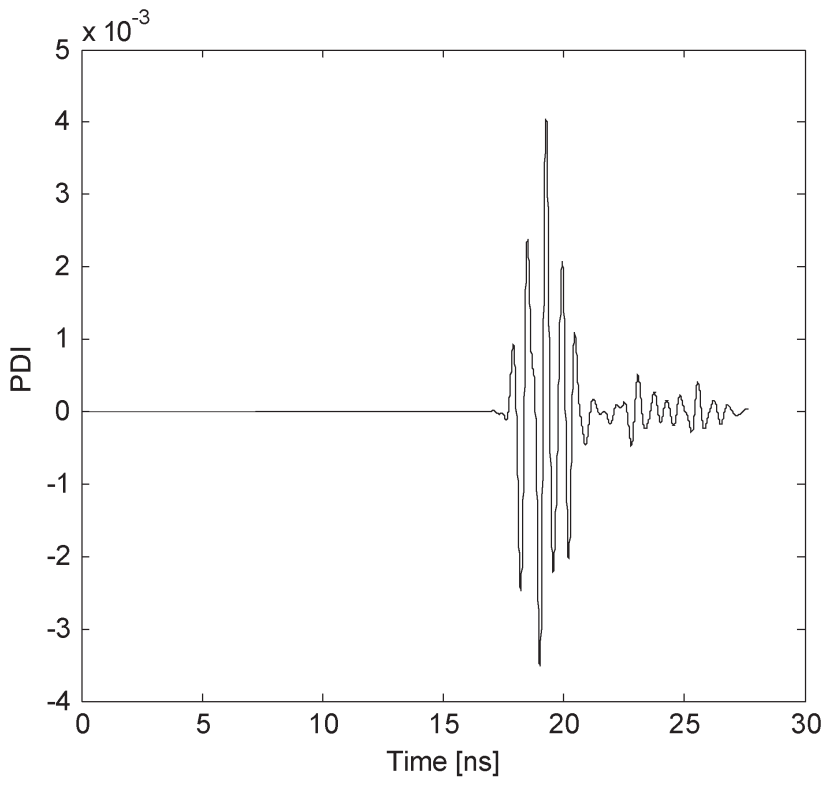

(b)

Fig. 1. Conventional PDI technique as applied to the numerically computed scattered fields from a cylindrical target behind a concrete wall. (a) Copolar component of the scattered field. (b) PDI signal.

compared to the "background." As done in [21], in this paper, we use criteria such as maximum multiplicative coefficients in the PC outputs and the maximum variances for the second PC to determine the optimal channels. Simulation and experimental results in the visible regime confirmed that the new APDI technique outperforms the original nonadaptive PDI technique in most situations [21].

In the present work, we utilize and adjust this technique for the TWMI applications. Here, we first present a short overview of the algorithm, emphasizing only the particular points relevant to the TWMI problems. The APDI algorithm presented here is to be eventually implemented in the TWMI system, and therefore, as much as possible, the discussion of the technique is related to some relevant scenarios. In addition, we present an alternative algorithm, named transient APDI (or TAPDI) that extends the frequency-domain APDI to time-domain radar imaging. The proposed APDI and TAPDI techniques are particularly useful in mitigating the potential masking effects of walls as well as detecting motion, when Doppler techniques are not applicable. We note that, even though the focus of this paper is mainly on the applications of these algorithms to motion detection and sensing, nevertheless, throughout this paper, we have used the phrase "polarization difference imaging" as the names of these algorithms in order to adhere to their original development and terminology in optical imaging. Some of the results presented in this paper originally appeared in our earlier work [23].

\section{OVERVIEW OF THE APDI AlgorithM}

It is known that a target with distinct polarization properties, such as a cylinder, has a preferential polarization in the scattered field. Thus, we introduce the metrics for PDI as

$$
P D I=\left(\left|E_{\theta \theta}\right|^{2}+\left|E_{\theta \phi}\right|^{2}\right)-\left(\left|E_{\phi \phi}\right|^{2}+\left|E_{\phi \theta}\right|^{2}\right)
$$

where $E_{\theta \theta}$ and $E_{\theta \phi}$ are the co- and cross-polarized scattered electric field by an $E_{\theta}$-polarized incident field and $E_{\phi \theta}$ and $E_{\phi \phi}$ by an $E_{\phi}$-polarized incident field, respectively. We note that one may use other alternative expressions, e.g., $P D I=$ $E_{\theta \theta}(t)-E_{\phi \phi}(t)$. It is obvious that $P D I=0$ for a symmetric polarization-insensitive object (e.g., a sphere) and $P D I \neq 0$ for a nonsymmetric object (e.g., a cylinder). An example for a conducting cylinder, with diameter of $10 \mathrm{~cm}$ and length of $70 \mathrm{~cm}$, at a distance of $1 \mathrm{~m}$ behind a homogeneous concrete wall with a thickness of 8 in with a dielectric constant of $\varepsilon_{r}=7.66 \varepsilon_{0}$ and conductivity of $\sigma=0.06 \mathrm{~S} / \mathrm{m}$, is shown in Fig. 1, where it is shown that the aforementioned simple PDI metric may be used to remove the wall effects.

The general idea behind the APDI technique, which is an extension of the simple PDI concept, is that the imaging system can be "adapted" to the observation scene in the absence of the target by utilizing the polarization statistics of the scene in the previous look, i.e., on the polarization information from the background (i.e., "nontarget") scene. This adaptation makes the changes in the scene, e.g., appearance/disappearance of objects and/or changes in the mutual orientation of the objects, more pronounced and noticeable to the adapted imaging system. Thus, for TWMI applications, we assume that, prior to the time of target detection, the original background scene is considered a nontarget scene or a background. Then, when the target becomes present or its status changes, the scene is assumed to become a target scene. Our goal in using APDI for this scenario is to be able to detect any changes that occur between the nontarget (i.e., background) and the target (background plus target) scenes. We note that, in some scenarios, such as motion detection, simple background subtraction may not solve the problem, due to changes in the scene and possible changes in the imaging system look angle. In order to apply our technique, the amplitudes and phases of the two orthogonal components of a scattered electric field, i.e., co- and cross-polarized 


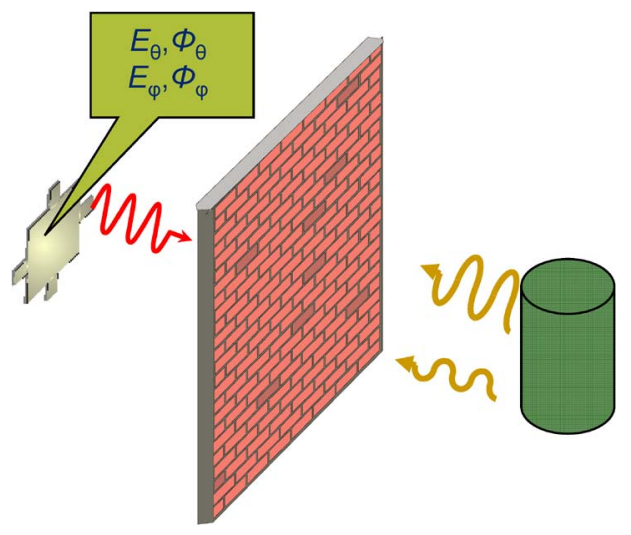

Fig. 2. Target behind wall. The scattered fields are collected on the receive array aperture for APDI implementation.

components, are required at each element of the receiving antenna array.

Alternatively, one may consider the background and target scenes to be the target when it is illuminated by horizontally and vertically polarized incident waves, respectively. This alternative scheme is particularly attractive for the imaging of static scenes in TWMI, when one may not have access to the empty scene with only the wall present.

The APDI algorithm may be subdivided into several steps. First, when the background scene is illuminated with a horizontally or vertically polarized monochromatic incident wave, the amplitudes and phases of two orthogonal polarized components of electric fields scattered from the observed scene, i.e., $\left|E_{\theta}\right|$ and $\Phi_{\theta}$, and $\left|E_{\varphi}\right|$ and $\Phi_{\phi}$, are collected at each receiving point in the plane of detection, where $\left|E_{\theta}\right|$ and $\Phi_{\theta}$ are the amplitude and phase of $\theta$ component of the scattered electric field, respectively, and $\left|E_{\varphi}\right|$ and $\Phi_{\varphi}$ are those of the $\varphi$ component (see Fig. 2), respectively. It should be mentioned that, unlike the optical imaging scenarios, in the microwave imaging due to the availability of phase information, all four Stokes parameters of the observing scene can be computed [25], and phase information can be utilized.

Next, from the knowledge of amplitudes and phases of scattered field components at the receiving points, we can synthesize the outputs of the two "virtual channels" (or "receiving antennas") that can be oriented at arbitrary angles $\psi_{1}$ and $\psi_{2}$. Those outputs, given as $F_{b}^{1}$ and $F_{b}^{2}$, can be expressed as follows:

$$
\begin{aligned}
& F_{b}^{1}\left(\psi_{1}\right)=|| E_{\varphi}\left|e^{j \Phi_{\varphi}} \cos \psi_{1}+\right| E_{\theta}\left|e^{j \Phi_{\theta}} \sin \psi_{1}\right| \\
& F_{b}^{2}\left(\psi_{2}\right)=|| E_{\varphi}\left|e^{j \Phi_{\varphi}} \cos \psi_{2}+\right| E_{\theta}\left|e^{j \Phi_{\theta}} \sin \psi_{2}\right|
\end{aligned}
$$

with $0 \leq \psi_{1}$ and $\psi_{2} \leq 180^{\circ}$. In our notation, the subscript $b$ stands for the background scene. These signals represent the field scattered from the object of interest, and a set of optimal signals can then be obtained as a linear combination of $F_{b}^{1}$ and $F_{b}^{2}$ with certain weighting coefficients. In order to select appropriate channels, i.e., the appropriate angles of orientation for the receiving antennas, to achieve the optimal signals, the well-known algorithm of PCA [25] is utilized. To this end, $M \times N$ receiving points are considered in the plane of detection, and at each of these points, the two signals $F_{b}^{1}\left(\psi_{1}\right)$ and $F_{b}^{2}\left(\psi_{2}\right)$ are evaluated. According to PCA, the covariance matrix for such an arbitrary pair of signals is defined in (3), shown at the bottom of the page, where $W[F]=$ $(1 / M N) \sum_{m=1}^{M} \sum_{n=1}^{N} F\left(x_{m}, y_{n}\right)$ is the mean value taken over the ensemble of receiving points in the detection plane and $F$ represents $F_{b}^{1}$ and $F_{b}^{2}$ or their product as required in the expression for $C\left(\psi_{1}, \psi_{2}\right)$. Once the eigenvalues $\left(\lambda_{1}, \lambda_{2}\right)$ and the eigenvectors of the covariance matrix $C$ are determined, the transformation matrix, which has the eigenvectors as its rows, is formed as follows:

$$
T\left(\psi_{1}, \psi_{2}\right)=\left[\begin{array}{cc}
-\beta\left(\psi_{1}, \psi_{2}\right) & \alpha\left(\psi_{1}, \psi_{2}\right) \\
\alpha\left(\psi_{1}, \psi_{2}\right) & \beta\left(\psi_{1}, \psi_{2}\right)
\end{array}\right]
$$

As is normally done, the order of the eigenvectors is such that the first eigenvector corresponds to the largest eigenvalue. The PCA is performed on signals $F_{b}^{1}\left(\psi_{1}\right)$ and $F_{b}^{2}\left(\psi_{2}\right)$, corresponding to all possible combinations of the angle of orientation of the receiving antenna (i.e., all possible values of $\psi_{1}$ and $\psi_{2}$ from 0 to $180^{\circ}$ ). From the PCA, we find four parameters, namely, the two elements forming the eigenvectors [as shown in (4)] acting as weighting coefficients $\alpha$ and $\beta$ (also called "adaptive" multiplicative coefficients) and the two eigenvalues $\lambda_{1}$ and $\lambda_{2}$, which are all functions of $\psi_{1}$ and $\psi_{2}$. Based on our previous work in optical imaging [21], an "optimal" pair of angles, $\psi_{1}^{\mathrm{opt}}$ and $\psi_{2}^{\text {opt }}$, is selected as a pair that corresponds to the peak values of the weighting coefficient $\alpha$ (or $\beta$ ), i.e.,

$$
\alpha^{\mathrm{opt}}=\alpha\left(\psi_{1}^{\mathrm{opt}}, \psi_{2}^{\mathrm{opt}}\right) \quad \beta^{\mathrm{opt}}=\beta\left(\psi_{1}^{\mathrm{opt}}, \psi_{2}^{\mathrm{opt}}\right)
$$

For a detailed discussion of the issues on selecting the optimal coefficients in the context of optical imaging, the reader is referred to [21].

In the next step, we consider the target scene that is illuminated by the same incident wave. The amplitudes and phases of the orthogonally polarized components of the scattered field from the target scene are collected, and then, the outputs from the two virtual "channels," corresponding to a pair of optimal angles that were previously obtained from the background scene, are synthesized following the analogous expression given in (2). Let us denote these outputs for the target scene as $F_{t}^{1}$ and $F_{t}^{2}$. Here, the index $t$ stands for the target scene.

$\mathrm{PCs}$ of the scene are now created as follows:

$$
\left[\begin{array}{l}
P C_{1} \\
P C_{2}
\end{array}\right]=\left(\begin{array}{cc}
-\beta^{\mathrm{opt}} & \alpha^{\mathrm{opt}} \\
\alpha^{\mathrm{opt}} & \beta^{\mathrm{opt}}
\end{array}\right)\left[\begin{array}{l}
F_{t}\left(\psi_{1}^{\mathrm{opt}}\right) \\
F_{t}\left(\psi_{2}^{\mathrm{opt}}\right)
\end{array}\right]
$$

$$
C\left(\psi_{1}, \psi_{2}\right)=\left[\begin{array}{cc}
W\left[F_{b}^{1} F_{b}^{1}\right]-W^{2}\left[F_{b}^{1}\right] & W\left[F_{b}^{1} F_{b}^{2}\right]-W\left[F_{b}^{1}\right] W\left[F_{b}^{2}\right] \\
W\left[F_{b}^{1} F_{b}^{2}\right]-W\left[F_{b}^{1}\right] W\left[F_{b}^{2}\right] & W\left[F_{b}^{2} F_{b}^{2}\right]-W^{2}\left[F_{b}^{2}\right]
\end{array}\right]
$$




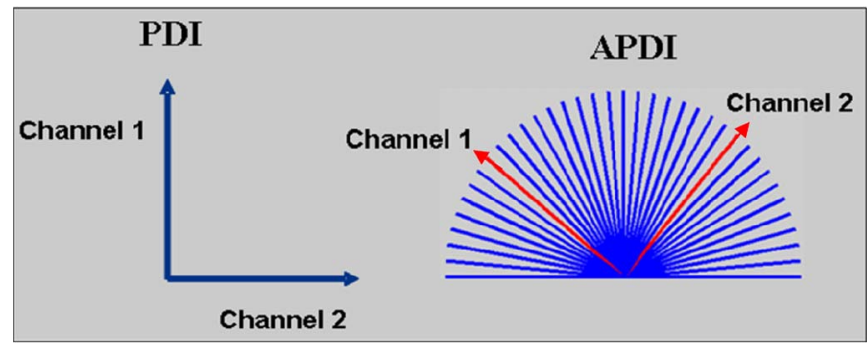

Fig. 3. Conventional PDI with two fixed channels and APDI with two optimum channels adapted to the polarization statistics of the scene.

where the elements of the matrix are the coefficients obtained from the background scene. As will be seen later in the next section, the $\mathrm{PC}_{2}$ image can provide us with better target-tobackground distinction. Fig. 3 shows the comparison between two fixed vertical and horizontal channels in the conventional PDI with the aforementioned "optimum" channels in APDI.

\section{APPLICATIONS OF APDI TECHNIQUE IN TARget Detection Problems}

In this section, we present several simulations of the APDI technique for the detection of changes in the orientations of the objects within a scene. In order to maintain the consistency, the target object was kept the same in the course of all the different through-wall scenarios given in this section. Here, we present several scene environments containing the same target, namely, a simple model of a human torso and arm in order to compare and test the performance of the algorithm.

\section{A. Detection of Changes in the Orientation of the Human Arm Behind Wall}

One of the important problems to be considered in target detection applications is the detection of a human's body motion or changes in its orientation behind a lossy dielectric wall. Here, we discuss a simplified geometry of this case that may nevertheless be a representative for the potential applications of our algorithm.

The geometry of the problem considered is shown in Fig. 4. The object of observation consists of the two circular cylinders attached to each other at one fixed point. The larger cylinder represents a part of the human torso, and the smaller cylinder models a part of the human arm. The material parameters of these cylinders are chosen to be somewhat similar to those of human muscle, i.e., the permittivity $\varepsilon=52 \varepsilon_{0}$, where $\varepsilon_{0}$ is the permittivity of free space, and conductivity $\sigma=1.2 \mathrm{~S} / \mathrm{m}$. An incident plane wave with the frequency $f=2.0 \mathrm{GHz}$ propagates in the $+y$ direction $\left(\varphi=90^{\circ}, \theta=90^{\circ}\right)$ along the negative $y$ axis toward the $-y$ direction (see Fig. 4). The objects are located a meter away from an infinitely extent wall, behind which the plane of receiving antenna array is positioned. As typical examples for walls, we consider homogeneous concrete and wooden walls. The thickness of the wall in both cases is $10 \mathrm{~cm}$. The dielectric constant and conductivity for the concrete and wooden walls are $\varepsilon_{r}=6 \varepsilon_{0}$ and $\sigma=0.06 \mathrm{~S} / \mathrm{m}$, and $\varepsilon_{r}=3 \varepsilon_{0}$ and $\sigma=0.006 \mathrm{~S} / \mathrm{m}$, respectively.

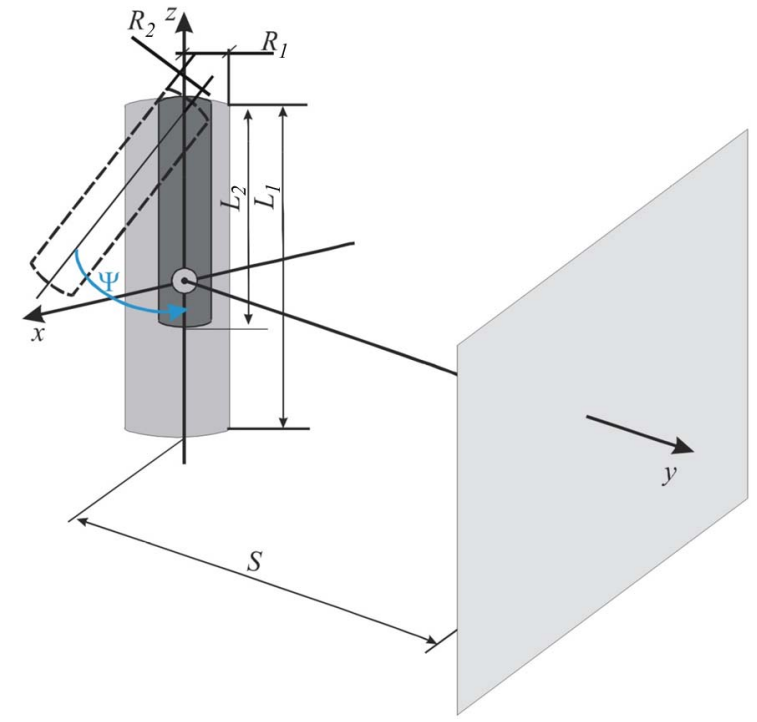

Fig. 4. Model of human arm and torso located behind the wall, $L_{1}=85 \mathrm{~cm}$, $L_{2}=45 \mathrm{~cm}, R_{1}=10 \mathrm{~cm}, R_{2}=5 \mathrm{~cm}$, and $S=1 \mathrm{~m}$. The wall is $10 \mathrm{~cm}$ thick and made from concrete or wood.

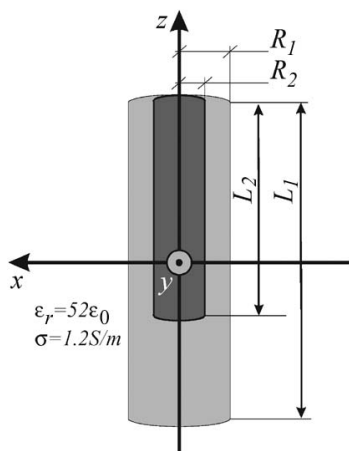

(a)

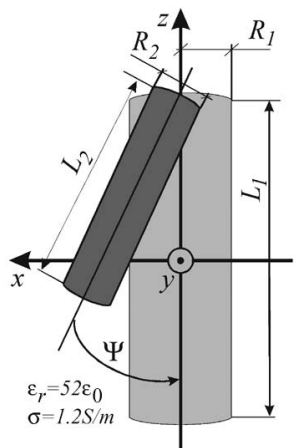

(b)
Fig. 5. Model of human arm and torso. (a) Nontarget, i.e., background scene. (b) Target scene.

The initial position of the arm (i.e., the smaller cylinder) along the torso (i.e., the larger cylinder) is considered as the first background scene. In our model, this case is represented by the two cylinders parallel with each other and having their upper edges at the same level (see panel (a) in Fig. 5). Starting from this initial situation, the position of the smaller cylinder is changed by rotating the arm with respect to the torso. The next position of the human arm, which is different from the initial orientation, is considered as the first target scene. Therefore, the goal is to distinguish two scenarios of background versus target cases (i.e., between the case where two cylinders are parallel versus the case where the smaller cylinder is oriented differently (see panel (b) in Fig. 5). These scenes basically model the motion of the human arm through changes in its orientation. Here, we present results when angle $\Psi$ between the axis of the arm and torso is $0^{\circ}, 30^{\circ}, 60^{\circ}$, or $90^{\circ}$. Although, in our study, both polarizations of the incident wave are considered, in this paper, for the sake of brevity, we present results corresponding to the incident $\theta$-polarized wave only, where the plane of polarization of the incident wave coincides with the plane of the human torso. 

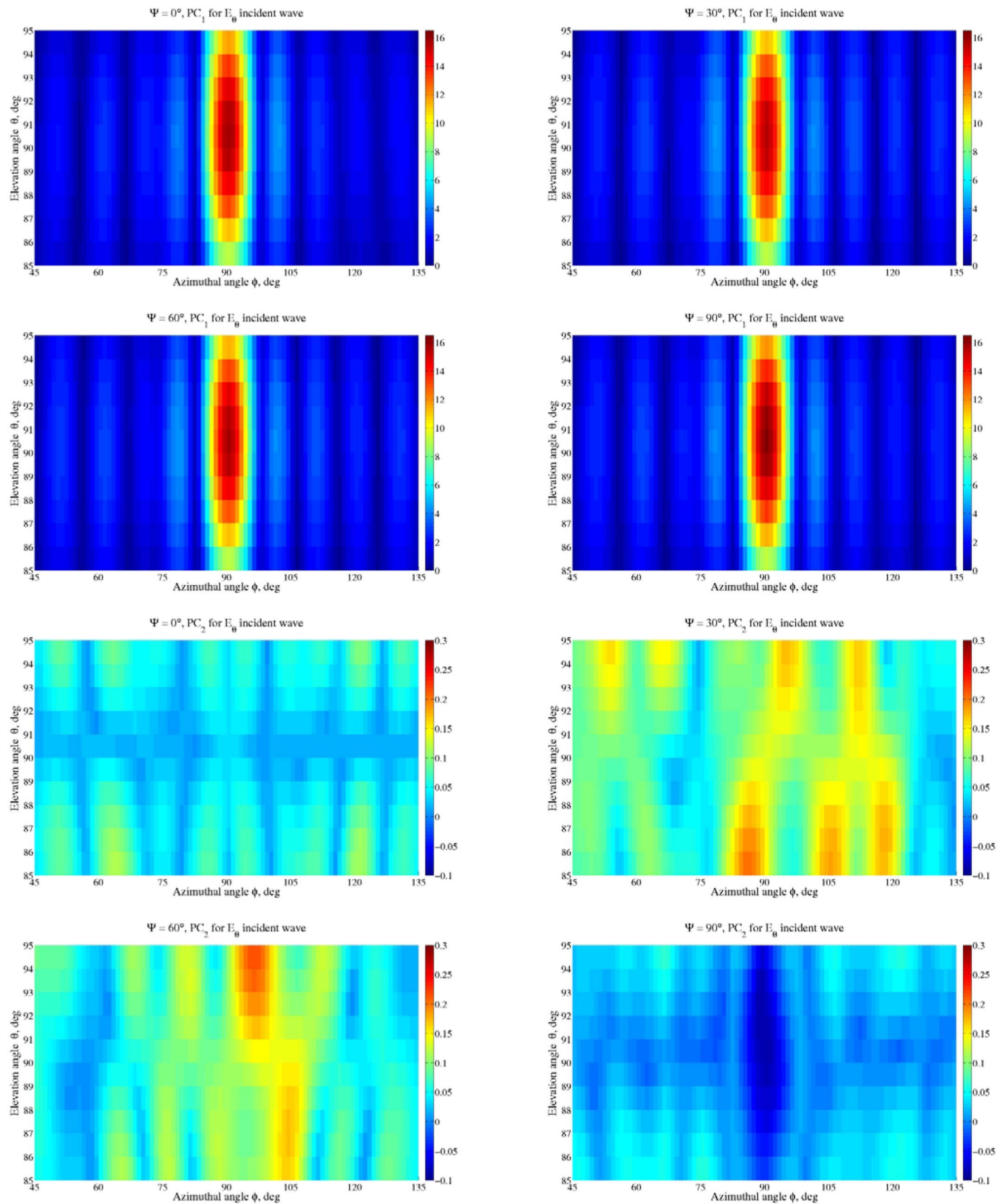

Fig. 6. Marching-scheme variation of the PC signals for different orientations of human arm behind a concrete wall. $P C_{1}$ (first and second rows) and $P C_{2}$ (third and fourth rows) are plotted as a function of $\theta$ and $\phi$ in the plane of the receiving array for different angles $\Psi$ of the arm.

The finite-difference time-domain (FDTD) simulations were performed using Remcom XFDTD commercial software package, which is based on the well-known FDTD technique (see, e.g., [24]). The amplitudes and phases of the scattered electric field were collected in the far zone in the sector around the normal to the wall $\left(\theta=\phi=90^{\circ}\right)$, namely, $85^{\circ} \leq \theta \leq 95^{\circ}$ 


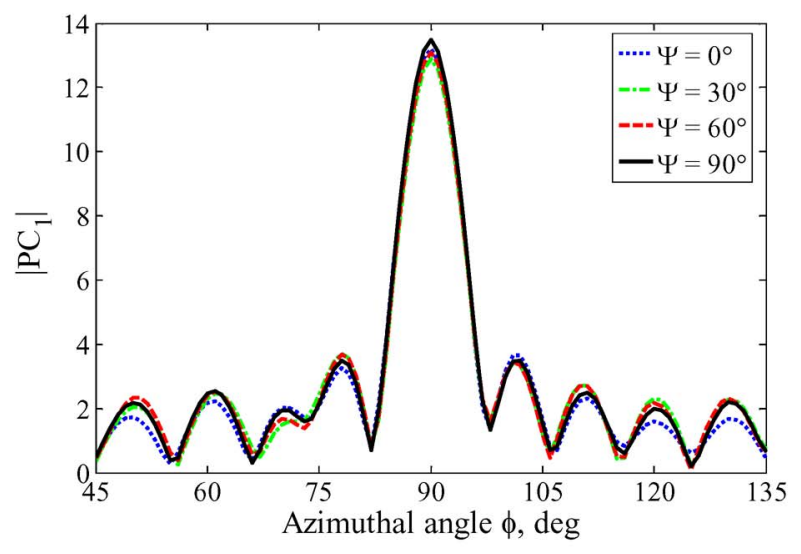

(a)

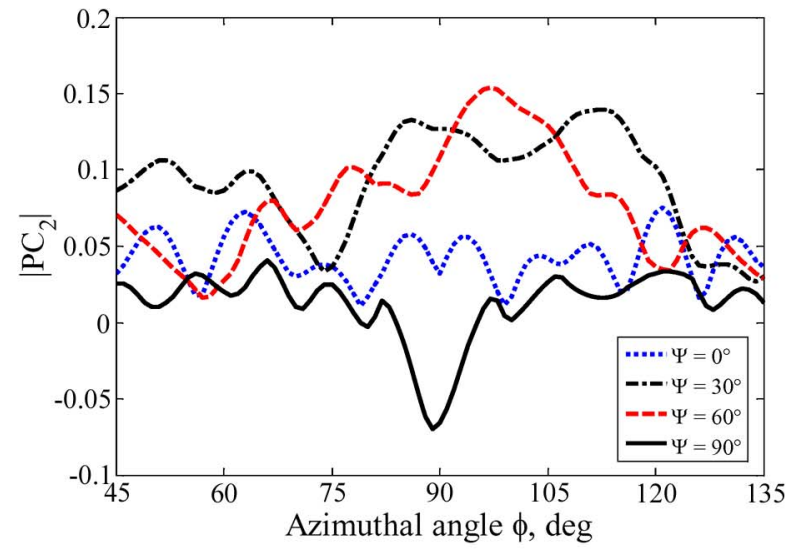

(b)

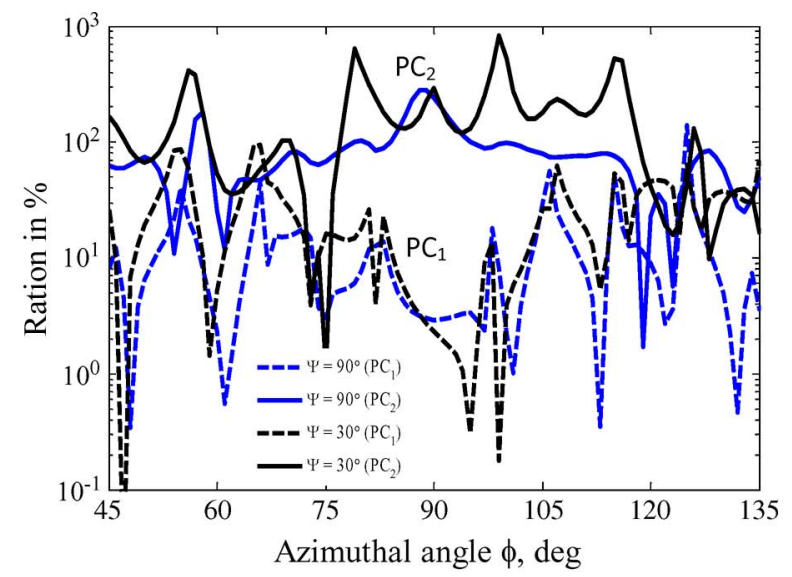

(c)

Fig. 7. PC signals versus azimuth angle $\phi$ averaged over the elevation angle $\theta$ for different relative orientations of the human arm behind a concrete wall. (a) Plot of $P C_{1}$. (b) Plot of $P C_{2}$. (c) Plot of the parameter $r$ given in (7) for both $P C_{1}$ and $P C_{2}$.

and $45^{\circ} \leq \phi \leq 135^{\circ}$. Further data processing was performed in the MathWorks MATLAB software package.

In Fig. 6, we show the first and second principal components of the scene, i.e., $P C_{1}$ and $P C_{2}$, for the objects behind the concrete wall; these are shown as 2-D plots with the $x$ and $y$ axes being the azimuth angle $\phi$ and the elevation angle $\theta$, respectively. The plots are shown in the same color-map scale within its own group (i.e., the scale is the same only within the same $\mathrm{PC}$ group, i.e., $P C_{1}$ or $P C_{2}$ ). All $P C_{1}$ and $P C_{2}$ are presented in the same far field angular ranges of $\theta$ and $\phi$. It is important to point out that neither of the PCs shown here are actual "images" of the scene. These are values of $P C_{1}$ and $P C_{2}$ at the points on the plane of the receiving array as functions of azimuth and elevation angles. No beamforming algorithms have been applied here. The figures shown here are the so-called "synthesized" signals, which manifest certain changes that have occurred in the scene.

Each panel in Fig. 6 shows the situation, where, for a given position of the arm, the background scene is assumed to be its previous position. Such a case may be considered as a surveillance-type application. Thus, for the target scene where the angle between the arm and torso is $\Psi=30^{\circ}$, the background is, for example, the scene with $\Psi=0^{\circ}$ in Fig. 6(a), and subsequently, the scene with $\Psi=30^{\circ}$ becomes the background for the $\Psi=60^{\circ}$ and so on; we call this a marching scheme. In each case, the target scene is analyzed using the polarization statistics of its corresponding background scene.

Comparing the panels in Fig. 6, one may notice that the shape of the first PC is very similar for all orientations of the human arm and is dominated mostly by the wall, whereas the distribution of $P C_{2}$ shows significant changes as the orientation of the arm varies. In other words, $P C_{2}$ seems to have effectively mitigated the wall effect and is mainly dominated by the changes that have occurred behind the wall. In order to better demonstrate this, the PC signals, averaged over the elevation angle $\theta$, are shown in Fig. 7 versus the azimuth angle $\phi$. We note that, in general, the plots of both PCs may be diverse enough to suggest changes in the scene. Thus, it is important to evaluate the component with the more significant relative change. Such a component may better serve for target detection purposes. In order to determine this, we compute the following parameter for both PCs, namely:

$$
r=100 \cdot \frac{\left\langle P C_{i}^{T}\right\rangle-\left\langle P C_{i}^{\mathrm{BG}}\right\rangle}{\left\langle P C_{i}^{\mathrm{BG}}\right\rangle} \quad \text { (in percent) }
$$

where $i=1,2$ and $\left\langle P C^{T}\right\rangle$ and $\left\langle P C^{\mathrm{BG}}\right\rangle$ are the PC signals of the target and background scenes, respectively, averaged over the elevation angle $\theta$. Comparing the distributions of $r$ over the azimuth angle for both principal components in Fig. 7(c), 

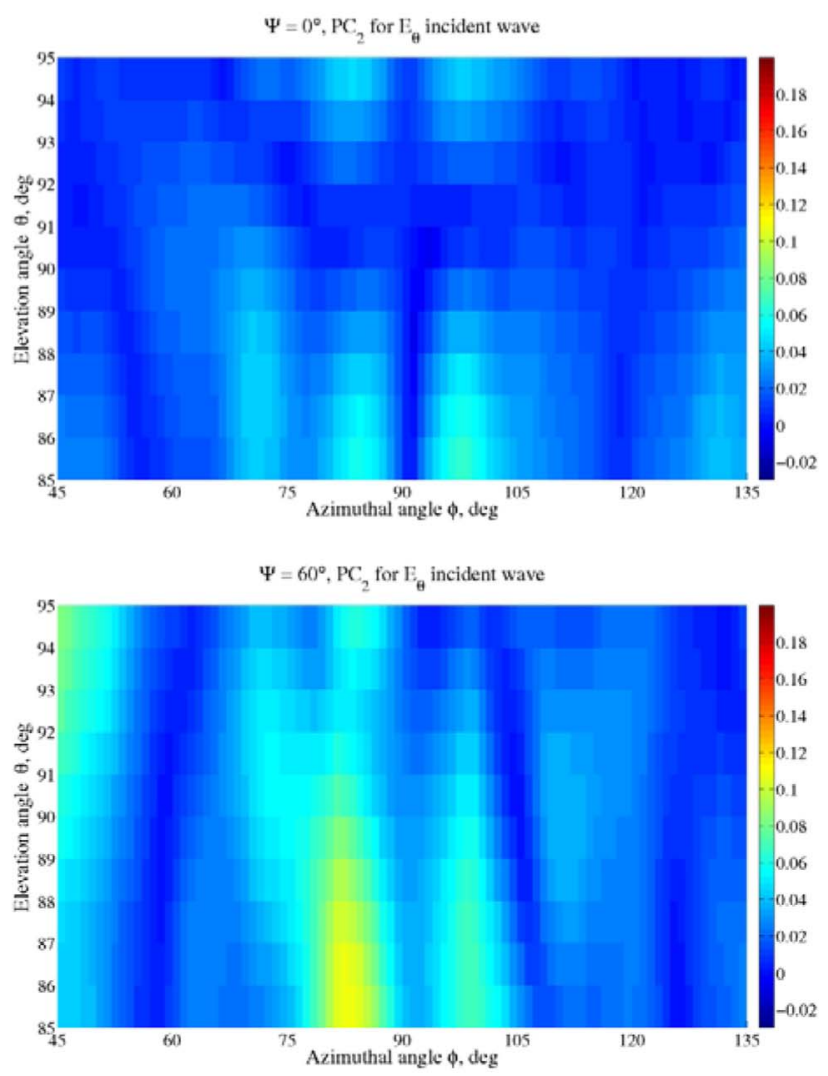
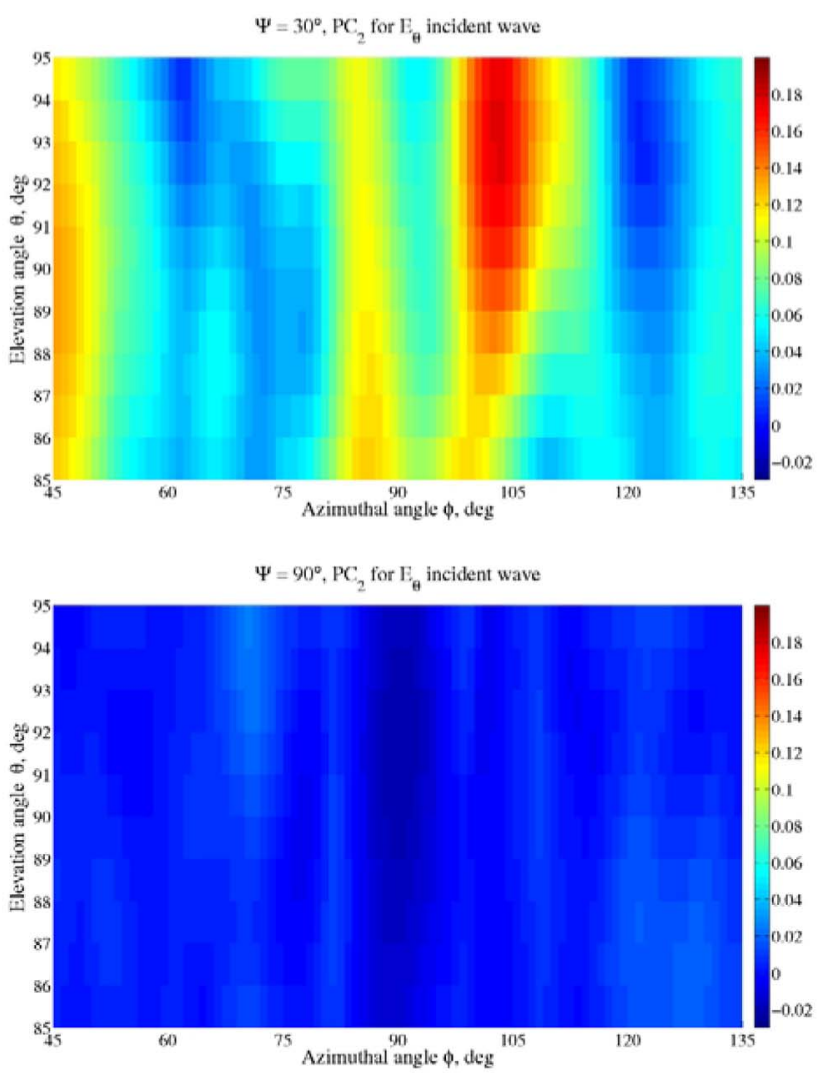

Fig. 8. Marching-scheme variation of the $P C_{2}$ signals for different orientations of the human arm behind a wooden wall. $P C_{2}$ is plotted as a function of $\theta$ and $\phi$ in the plane of the receiving array for different angles $\Psi$ of the arm.

one can notice that variations in $P C_{2}$ are, by and large, much greater than those in $P C_{1}$ [compare solid and dashed lines in Fig. 7(c)], and therefore, $P C_{2}$ may provide useful information for the detection of changes in the scene of observation.

The $P C_{2}$ plots for the case of the wooden wall are shown in Fig. 8. The previously mentioned marching scheme is utilized here as well. The plots of $P C_{1}$ are very similar to those of the concrete wall and, therefore, not shown here. The plots of $P C_{2}$ are different both by shape and scale, which is due to the difference in the dielectric properties of the walls' materials, but, in general, the transition in the $P_{2}$ images may enable one to characterize the possible changes in the scene.

We have also applied the APDI technique to the aforementioned example in the absence of the wall and have found similar results. The corresponding plot of the $r$ value in (7) for this case is shown in Fig. 9.

It is important to study the susceptibility of the algorithm to the influence of noise. Using the free-space example of Fig. 9, we artificially added Gaussian noise to the numerically computed scattered fields. The noise variance was defined as, $\sigma^{2}=$ $\left(P_{\max } / 10^{S N R / 10}\right)$, where $P_{\max }$ is the maximum power of the received signals and $S N R$ is the signal-to-noise ratio. The results are shown in Fig. 10 for the $r$ parameter corresponding to $P C_{1}$ and $P C_{2}$ in (7) when the arm's orientation is $\Psi=30^{\circ}$. As one may notice, the performance of the algorithm is relatively stable in the presence of noise, and the $r$ value for $P C_{2}$ remains significantly larger than that of $P C_{1}$ at different noise levels.

Although the PC figures discussed in the previous examples are not as distinctive as those in optics [21], the use of both PCs

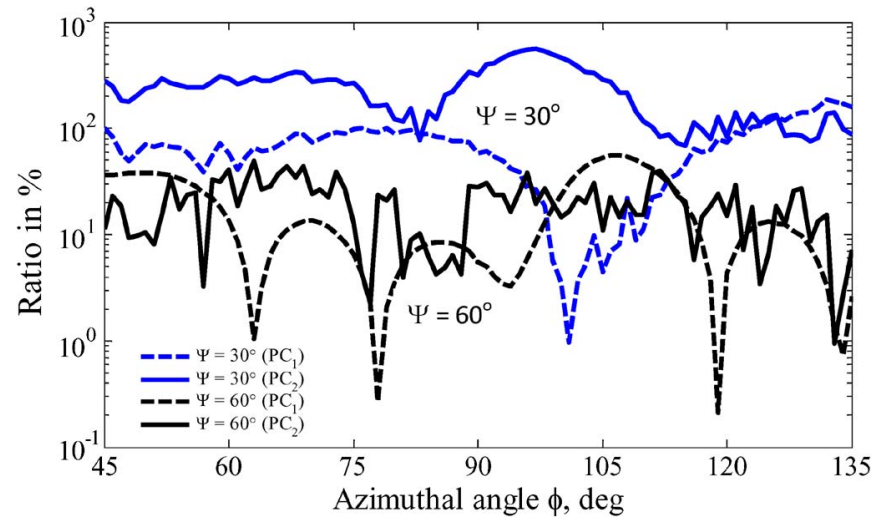

Fig. 9. Plots of the parameter $r$ given in (7) versus azimuth angle $\phi$ for both $P C_{1}$ and $P C_{2}$. The plots are shown for different relative orientations of the human arm in free space in the absence of the wall.

may provide additional information for the process of target detection. It is noteworthy that the changes in the angle $\Psi$ (i.e., motion of the arm with respect to the torso) cannot be detected by a conventional Doppler technique since in the aforementioned examples the incident wave travels in a direction normal to the direction of motion.

\section{B. Comparison of $\mathrm{PC}_{2}$ and Cross-Polar Field}

In the absence of a Doppler signal, the question on how the APDI technique compares with a standard polarimetric technique that employs the cross-polar components of the scattered 


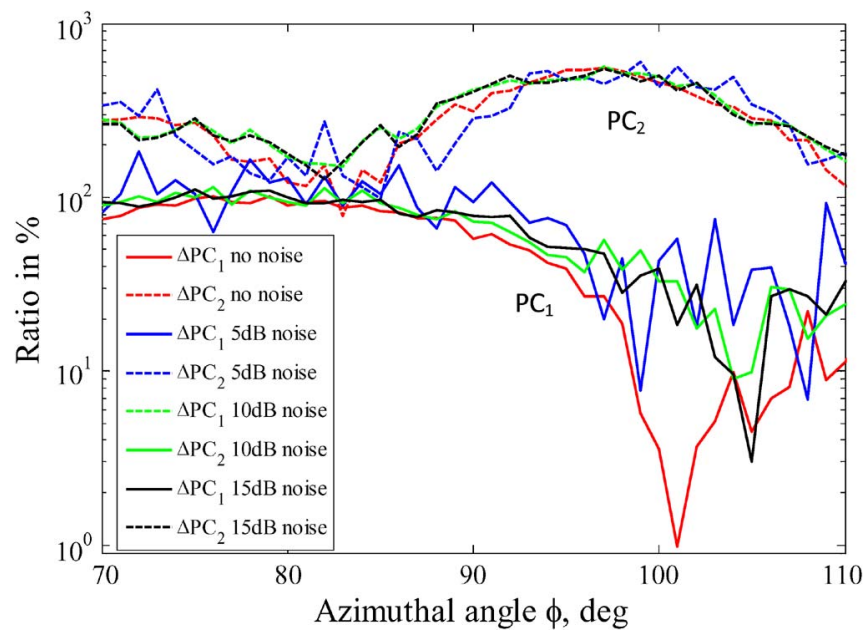

Fig. 10. Plots of the parameter $r$ given in (7) versus azimuth angle $\phi$ in the presence of Gaussian noise with different SNR values. The results are shown for the arm's orientation angle of $\Psi=30^{\circ}$.

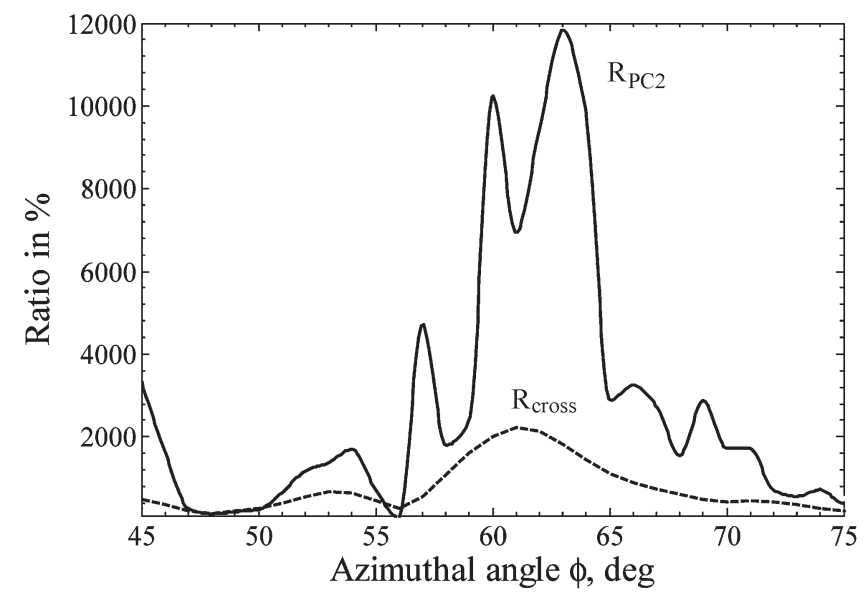

Fig. 11. Comparison of relative changes in $P C_{2}$ signal and the cross-polar component of the scattered field in the detection of a tilted conducting cylinder in free space.

field naturally arises. To answer this question, we have applied APDI to the simple problem of detecting changes in the orientation of a conducting cylinder of $150-\mathrm{cm}$ length and $10-\mathrm{cm}$ diameter. The background and the target scenes were assumed to be the cylinder when it was tilted at angles of $30^{\circ}$ and $60^{\circ}$, with respect to the $z$-axis, respectively. The incident wave was identical to that in the aforementioned examples and vertically polarized in the $z$-direction. Fig. 11 shows the relative changes in $P C_{2}$, as expressed by its $r$ value in (7), and the cross-polar scattered field as expressed by its corresponding $r$ value, $r=$ $100 \cdot\left\langle E_{\text {cross }}^{T}\right\rangle-\left\langle E_{\text {cross }}^{\mathrm{BG}}\right\rangle /\left\langle E_{\text {cross }}^{\mathrm{BG}}\right\rangle$. As can be seen, the $P C_{2}$ signal provides, by an order of magnitude, a significantly larger measure of the changes in the observed scene than that resulted from the cross-polar field.

\section{TAPDI ALGORITHM}

The APDI algorithm in Section II was developed for the case of a monochromatic incident wave. Here, the concept of APDI is extended to the transient case. The main difference between the two approaches is that, in TAPDI, the transient electromag- netic field scattered from the observed scene is processed within a certain temporal interval instead of the single-frequency case in APDI. In the transient case, we compute the PCs of the scene as functions of time, thus applying the APDI algorithm for the electromagnetic fields scattered from the scene in each temporal step. In addition, the phase information is not used, and only transient amplitudes of co- and cross-polarized components of the scattered field are collected in the plane of the receiving antenna.

Consider the scattered field from the scene of observation given by the transient amplitudes of co- and cross-polarized components, i.e., $\tilde{E}_{\theta}$ and $\tilde{E}_{\varphi}$, at the observation plane within a certain period of time. The field components may be obtained either in the course of measurements or from numerical simulations. Thus, at each temporal step $t_{n}=n \Delta t$, we have a pair of amplitudes at $m \times k$ spatial points in the observation plane. Here, without loss of generality, we consider $m=k$ (which corresponds to an $m \times m$ receive array in a typical TWMI setup). Providing these amplitudes are known, an output signal can be synthesized at any given orientation $\psi$ of the polarization plane of receiving antenna as

$$
\tilde{E}\left(t_{n}, \psi\right)=\tilde{E}_{\varphi}\left(t_{n}\right) \cos \psi+\tilde{E}_{\theta}\left(t_{n}\right) \sin \psi
$$

where index $n$ identifies the temporal frame, with $n=0$ corresponding to the moment when the transmitting antenna sends the pulse. The main assumption for the TAPDI is that we must initially have the two sets of co- and cross-polarized components of the electromagnetic field scattered from the scene within a certain interval of time for each polarization of incident field.

The procedure of the TAPDI algorithm can now be summarized in the following steps.

1) Generate, for each temporal frame of the "background scene," a set of pairs of signals according to (8), i.e., $\tilde{E}\left(t_{n}, \psi_{1}\right)$ and $\tilde{E}\left(t_{n}, \psi_{2}\right)$, where $\psi_{1}$ and $\psi_{2}$ are the angles of orientation of the polarization plane of any two receiving antennas (or virtual channels).

2) Apply the PCA algorithm to signals in each temporal frame and obtain all four adaptive parameters, i.e., eigenvalues $\lambda_{1}$ and $\lambda_{2}$ and coefficients $\alpha_{n}$ and $\beta_{n}$ as functions of angles $\left(\psi_{1}, \psi_{2}\right)$.

3) Find the optimal orientations of the receiving antennas in each temporal step $t_{n}$, i.e., $\psi_{1 n}^{\mathrm{opt}}=\psi_{1}^{\mathrm{opt}}\left(t_{n}\right)$ and $\psi_{2 n}^{\mathrm{opt}}=$ $\psi_{2}^{\mathrm{opt}}\left(t_{n}\right)$ with the corresponding optimal adaptive coefficients, i.e., $\alpha_{n}^{\mathrm{opt}}=\alpha\left(\psi_{1 n}^{\mathrm{opt}}, \psi_{2 n}^{\mathrm{opt}}\right)$ and $\beta_{n}^{\mathrm{opt}}\left(t_{n}\right)=$ $\beta\left(\psi_{1 n}^{\mathrm{opt}}, \psi_{2 n}^{\mathrm{opt}}\right)$.

4) Using the second temporal set of data, i.e., the "target scene," construct the two output signals for the optimal pair of angles $\psi_{1 n}^{\mathrm{opt}}$ and $\psi_{2 n}^{\mathrm{opt}}$ at each moment of time $t_{n}$ (for each temporal frame)

$$
\begin{aligned}
& P C_{1}\left(t_{n}\right)=-\beta_{n}^{\mathrm{opt}} \tilde{E}\left(t_{n}, \psi_{1 n}^{\mathrm{opt}}\right)+\alpha_{n}^{\mathrm{opt}} \tilde{E}\left(t_{n}, \psi_{2 n}^{\mathrm{opt}}\right) \\
& P C_{2}\left(t_{n}\right)=\alpha_{n}^{\mathrm{opt}} \tilde{E}\left(t_{n}, \psi_{1 n}^{\mathrm{opt}}\right)+\beta_{n}^{\mathrm{opt}} \tilde{E}\left(t_{n}, \psi_{2 n}^{\mathrm{opt}}\right)
\end{aligned}
$$

where $P C_{1}$ and $P C_{2}$ are the PCs of the scene at a given moment of time $t_{n}$ and $\tilde{E}\left(t_{n}, \psi\right)$ are the output signals from the two antennas, taken from the target scene data. 


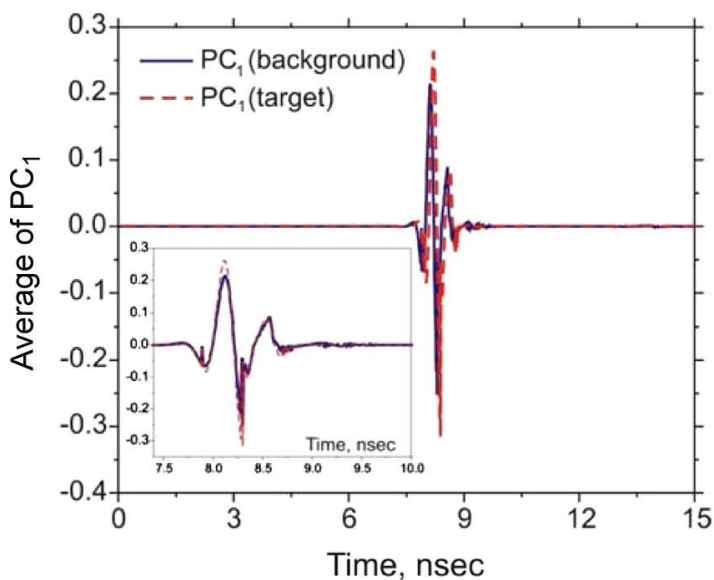

(a)

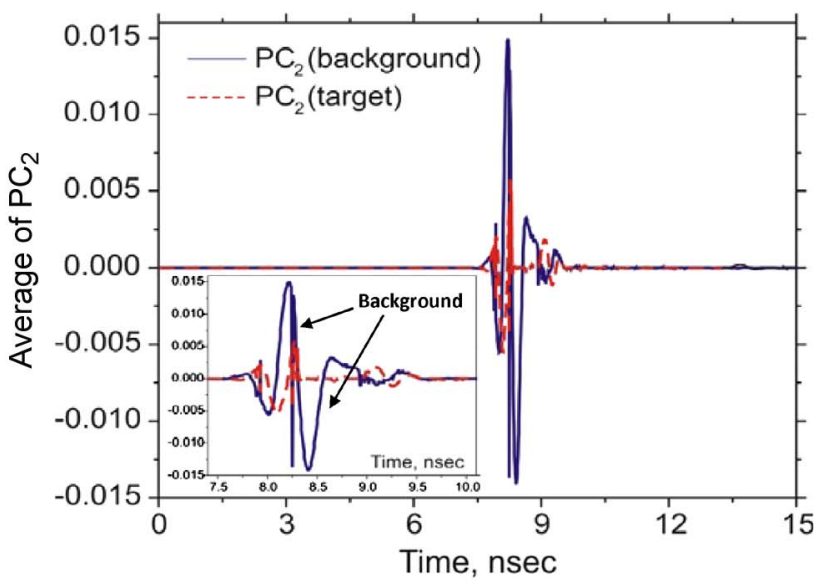

(b)

Fig. 12. Temporal distribution of PCs for the metal cylinder in the free space. (a) $P C_{1}$ signal. (b) $P C_{2}$ signal.

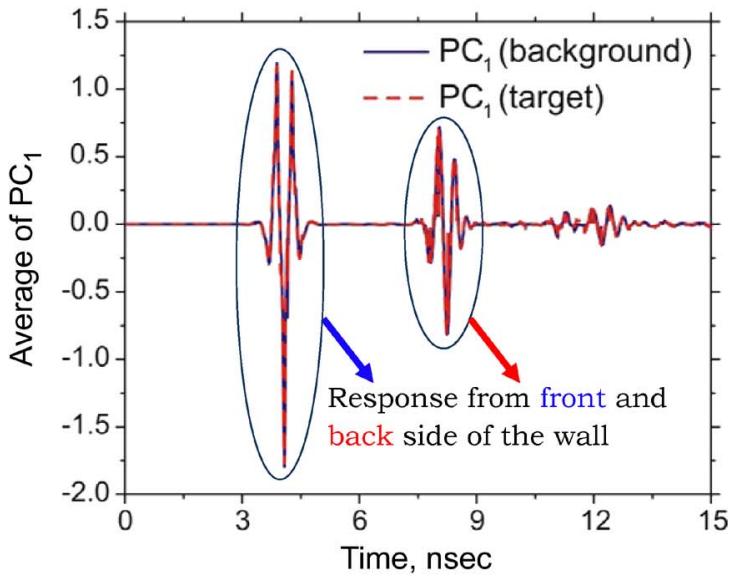

(a)

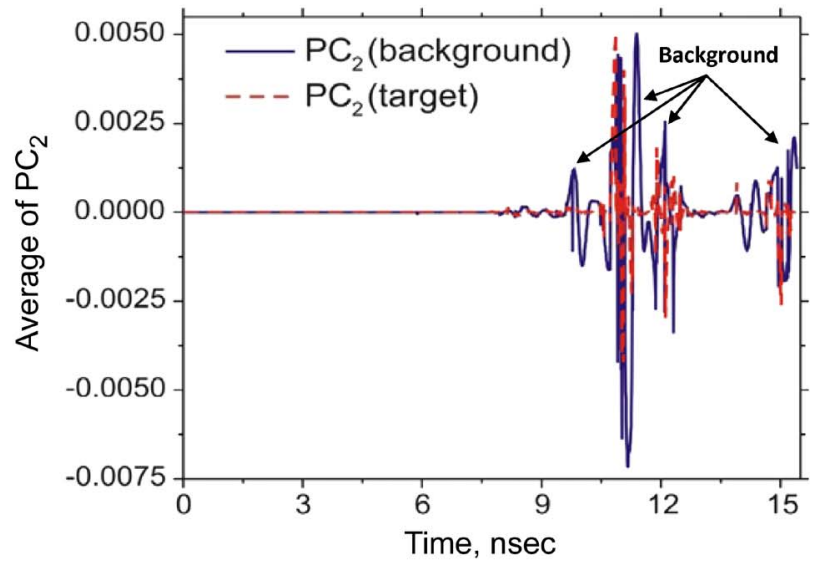

(b)

Fig. 13. Temporal distribution of PCs for the metal cylinder behind the concrete wall. (a) $P C_{1}$ signal. (b) $P C_{2}$ signal.

By observing temporal variations of the principal component signals $P C_{1}\left(t_{n}\right)$ and $P C_{2}\left(t_{n}\right)$, the presence of "target objects" or a change in it may, under certain circumstances, be detected.

\section{Application of TAPDi in TARget Detection}

\section{A. Detection of Target in Free Space and Behind}

\section{Homogeneous Walls}

In this section, the TAPDI technique is illustrated with an example on the detection of a canonical object both in the free space and in a through-wall scenario. The incident field is a $\theta$-polarized modulated Gaussian beam with center frequency of $2.5 \mathrm{GHz}$ and $-3-\mathrm{dB}$ levels at 2 and $3 \mathrm{GHz}$. The scattered field was simulated using the FDTD method.

As an example of a canonical target, a perfectly conducting cylinder of $150-\mathrm{cm}$ height and $10-\mathrm{cm}$ diameter was used. For the background scene, the cylinder was tilted with respect to the vertical position by $12^{\circ}$ in the plane parallel to the wall. The goal was to detect changes in the orientation of the cylinder in real time. The cylinder was located $1 \mathrm{~m}$ behind a finitesize concrete wall having a thickness, width, and height of

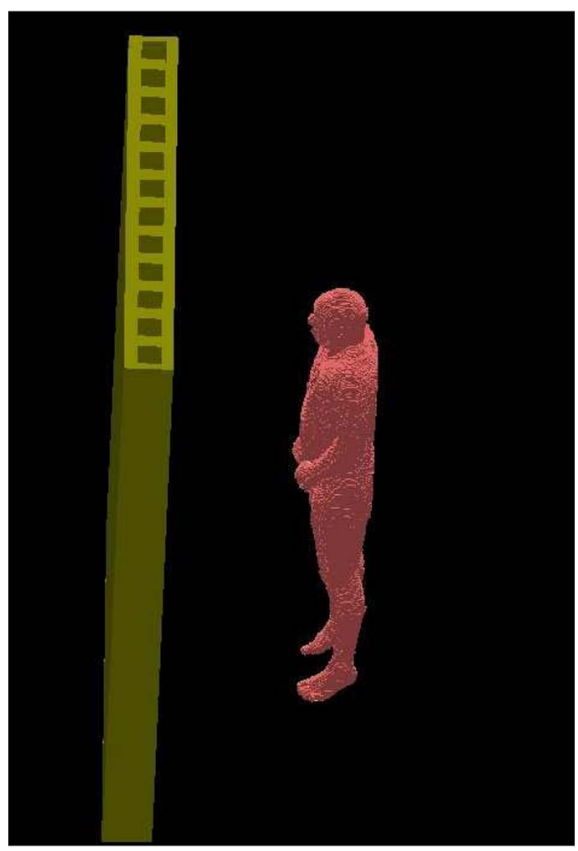

Fig. 14. High-fidelity frozen human model behind the cinder-block wall. 


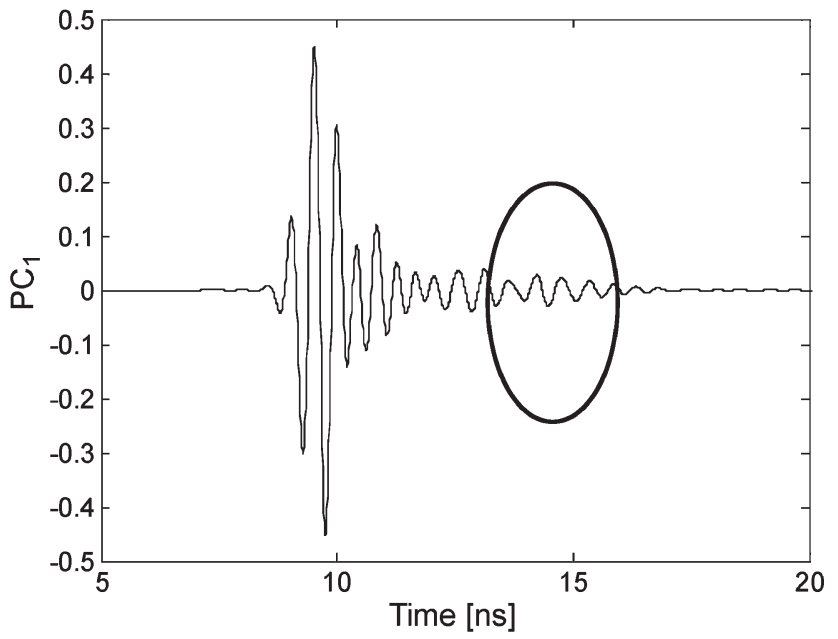

(a)

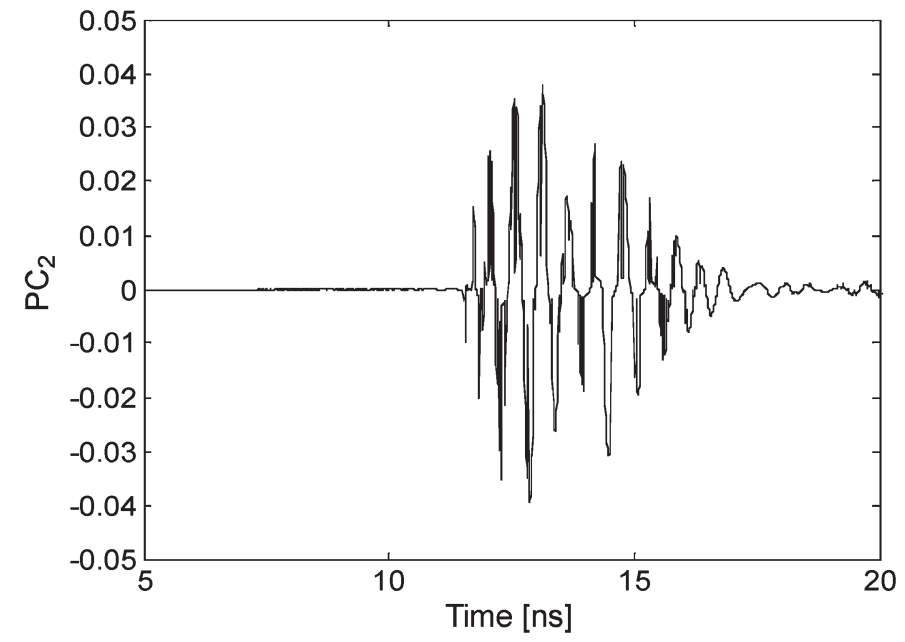

(b)

Fig. 15. PCs of the target scene for "frozen human" model behind the cinder-block wall. In the left panel, the oval encircles reflections from the human model. (a) $P C_{1}$ component. (b) $P C_{2}$ component.

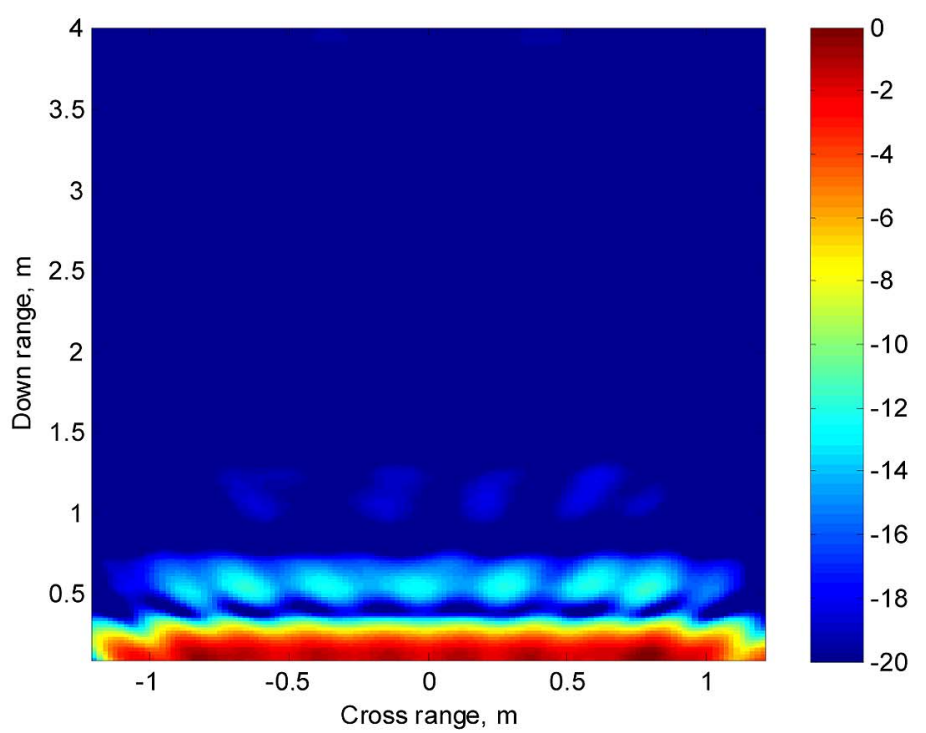

(a)

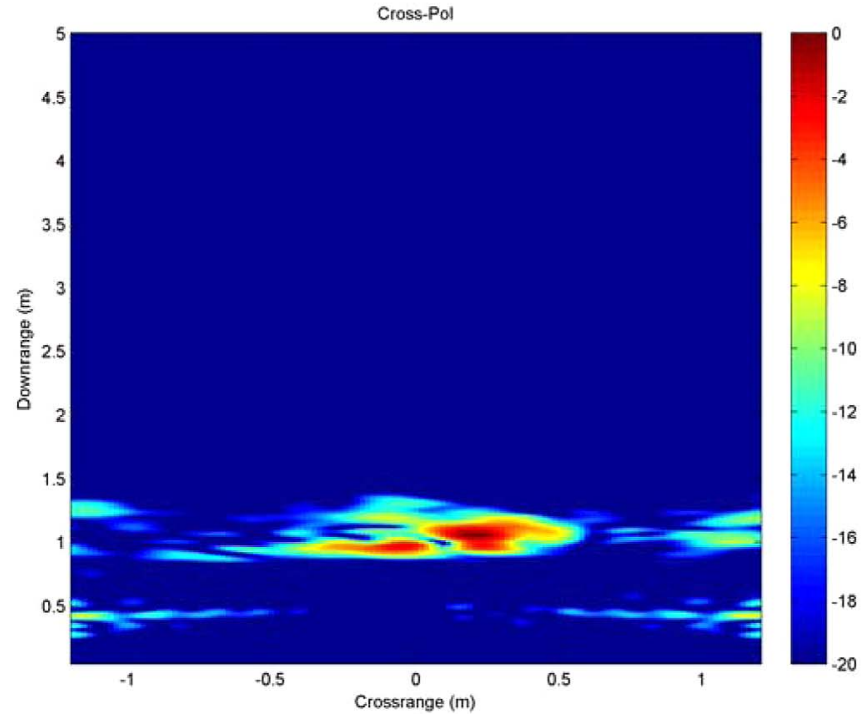

(b)

Fig. 16. Beamforming for the frozen human behind the cinder-block wall: (a) Copolarization image. (b) Cross-polarization image. The scene is illuminated with $E_{\theta}$-polarized incident field.

$17.8,80$, and $180 \mathrm{~cm}$, respectively. The scattered electric field components were collected in the range of $85^{\circ}-95^{\circ}$ for both $\theta$ and $\varphi$. For the sake of comparison, TAPDI was also applied to the same cylinder in the absence of the wall. In each case, the PCs of the background and target scenes were computed as functions of time.

The distributions of the PC signals for the no-wall case and the concrete wall are shown in Figs. 12 and 13, respectively. Every temporal frame is presented by a single point, which is the average value of corresponding PC data over 121 spatial points in the observation plane. As was already pointed out in the previous section, the $P C_{1}$ curves are similar for both the background and target cases. The temporal distributions of $P C_{2}$, however, are different for target and background cases, and unlike the $P C_{1}$ distributions, the reflections from the wall are eliminated for the concrete-wall case, as seen when comparing the left and right panels in Fig. 13.

In general, the TAPDI technique discussed previously is applicable to both homogeneous and inhomogeneous walls as long as the wall is not strongly polarized in the same direction as the target. In the following, we investigate the performance of the technique to the more challenging problem of target detection behind a cinder-block wall.

\section{B. Detection of Target Behind Cinder-Block Wall}

Periodic walls such as a hollow concrete (cinder) block wall pose difficult challenges in the detection of lossy targets such as humans. Since the cinder blocks form a heterogeneous wall with many air-wall interfaces, multiple reverberations and 
Bragg effects are present. These effects may mask the human signature and make it difficult to discern. In this section, we demonstrate the usefulness of the TAPDI algorithm to the detection of a human standing behind a cinder-block wall.

Fig. 14 shows a realistic model of a human standing behind a cinder-block wall. The full body human model used is the high-fidelity frozen male body commercially available from Remcom, Inc. The human's phantom consists of 5-mm cubical FDTD mesh cells and is composed of 23 different tissue types, each with its own dielectric properties. The cinder-block wall consists of $72(6 \times 12)$ standard cinder blocks. Each cinder block has dimensions of $40.62 \times 20.32 \times 20.3 \mathrm{~cm}$ and is made of cement with $\varepsilon_{r}=7.66 \varepsilon_{0}$ and $\sigma=0.06 \mathrm{~S} / \mathrm{m}$.

The scene is illuminated by a modulated Gaussian beam with the frequency band of $0.8-3.0 \mathrm{GHz}$, and the scattered field is collected in the far zone. For this example, TAPDI was applied differently by considering the background and target scenes both to be the human with the wall but with the scenes being illuminated by horizontally and vertically polarized incident waves, respectively. This alternative TAPDI technique may allow for the detection and imaging of static scenes in TWMI, without having to have access to the empty scene with only the wall present. The PCs of the target scene are shown in Fig. 15. As can be seen, even though the aforementioned TAPDI technique would be most effective for homogeneous walls, it is still able to mitigate, to a large extent, the cinderblock wall effects, resulting in a $P C_{2}$ signal, which, as compared to the $P C_{1}$ signal, is mainly due to the presence of the human behind the wall. We note, however, that some of the ringing due to the cinder-block wall and the interaction between the human's phantom and the wall are still present in $P C_{2}$.

The aforementioned results suggest that one could potentially use the $P C_{2}$ signal in a beamforming radar system to image the presence of the target behind the wall, even for a complex wall like cinder block, which is known to overshadow the target. Ideally, such imaging system requires a dual-polarized monostatic 2-D array, where $P_{2}$ is computed for each receiving antenna element due to a wave transmitted by a transmitting antenna at the same location in the array. The finite-difference time-domain simulation of such a system, however, is computationally very intensive and beyond the context of this paper. However, in order to demonstrate that simple polarization information of the scene may be used to reveal the presence of the human in the aforementioned example, we present the application of a standard delay-andsum beamformer to the received co- and cross-polarized fields when the scene is illuminated by a vertically polarized wave. The imaging system includes a linear 1-D receiving antenna array located at a distance of $1 \mathrm{~m}$ from the wall and consists of 31 elements spaced by half-wavelength apart at the center frequency. The results of the beamforming are shown in Fig. 16, where, as shown, the human target is overshadowed by the cinder-block wall in the copolarization image but is unmasked, with the wall effects to a large extent removed in the crosspolarization image. We note that the cross-polarized scattered fields play a significant role in the generation of the $P C_{2}$ signal in the TAPDI analysis.

\section{CONCLUSION}

In this paper, we employed the APDI and TAPDI techniques for certain TWMI applications. An overview of the APDI algorithm was presented, and several examples to illustrate some applications of the proposed method for detecting changes in the scene in both the absence and the presence of walls were given. The APDI algorithm was able to detect changes in the orientation of a human arm model with respect to a torso in both the no-wall and behind-the-wall scenarios. The algorithm maintains its performance even in a relatively complicated multipath environment and may help in detecting changes in the environment.

The frequency-domain APDI algorithm can be easily extended to the transient case in order to improve target detection, particularly motion detection, behind the wall in the time domain. Preliminary results on such a TAPDI algorithm were described for target detection in the through-wall microwave imaging applications. Numerical simulations with canonical and human-model objects demonstrated the potential of the TAPDI technique for target detection behind both homogeneous and periodic walls.

The proposed techniques may remove or reduce the masking effects of the wall and may be particularly useful in detecting motion when conventional Doppler techniques are not applicable. Future works include the experimental verification of the APDI and TAPDI techniques using measured results as well as the application of these techniques in more complicated TWMI environments.

\section{ACKNOWLEDGMENT}

The authors would like to thank Dr. F. Ahmad for fruitful discussions during the course of this research work. The content of the information does not necessarily reflect the position or the policy of the government, and no official endorsement should be inferred.

\section{REFERENCES}

[1] F. A. Sadjadi and A. Mahalanobis, "Target-adaptive polarimetric synthetic aperture radar target discrimination using maximum average correlation height filters," Appl. Opt., vol. 45, no. 13, pp. 3063-3070, May 2006.

[2] F. A. Sadjadi, "Adaptive polarimetric sensing for optimum radar signature classification using a genetic search algorithm," Appl. Opt., vol. 45, no. 22, pp. 5677-5685, Aug. 2006.

[3] F. A. Sadjadi, "Improved target classification using optimum polarimetric SAR signatures," IEEE Trans. Aerosp. Electron. Syst., vol. 38, no. 1, pp. 38-49, Jan. 2002.

[4] Radar Polarimetry for Geoscience Applications, F. T. Ulaby and C. Elachi, Eds. Norwood, MA: Artech House, 1990.

[5] L. M. Novak, M. C. Burl, and W. W. Irving, "Optimal polarimetric processing for enhanced target detection," IEEE Trans. Aerosp. Electron. Syst., vol. 29, no. 1, pp. 234-244, Jan. 1993.

[6] D. L. Evans, T. G. Farr, J. J. van Zyl, and H. A. Zebker, "Radar polarimetry: Analysis tools and applications," IEEE Trans. Geosci. Remote Sens., vol. 26, no. 6, pp. 774-789, Nov. 1998.

[7] E. Pottier and J. Saillard, "Optimal polarimetric detection of radar target in a slowly fluctuating environment of clutter," IEEE Aerosp. Electron. Syst. Mag., vol. 5, no. 11, pp. 4-9, Nov. 1990.

[8] D. Pastina, P. Lombardo, and T. Bucciarelli, "Adaptive polarimetric target detection with coherent radar. I. Detection against Gaussian background," IEEE Trans. Aerosp. Electron. Syst., vol. 37, no. 4, pp. 1194-1206, Oct. 2001. 
[9] D. Pastina, P. Lombardo, and T. Bucciarelli, "Adaptive polarimetric target detection with coherent radar. II. Detection against non-Gaussian background," IEEE Trans. Aerosp. Electron. Syst., vol. 37, no. 4, pp. 1207-1220, Oct. 2001.

[10] R. D. Chaney, M. C. Bud, and L. M. Novak, "On the performance of polarimetric target detection algorithms," IEEE Aerosp. Electron. Syst. Mag., vol. 5, no. 11, pp. 10-15, Nov. 1990.

[11] D. H. Dai, X. F. Wu, Y. Z. Li, X. S. Wang, and S. P. Xiao, "Polarization target detection algorithm based on instantaneous Stokes sub-vector," in Proc. IEEE Int. Symp. Microw., Antenna, Propag., EMC Technol. Wireless Commun., 2005, vol. 1, pp. 310-313.

[12] G. D. De Grandi, J.-S. Lee, and D. L. Schuler, "Target detection and texture segmentation in polarimetric SAR images using a wavelet frame: Theoretical aspects," IEEE Trans. Geosci. Remote Sens., vol. 45, no. 11, pp. 3437-3453, Nov. 2007.

[13] J.-C. Souyris, C. Henry, and F. Adragna, "On the use of complex SAR image spectral analysis for target detection: Assessment of polarimetry," IEEE Trans. Geosci. Remote Sens., vol. 41, no. 12, pp. 2725-2734, Dec. 2003.

[14] V. Komanduri, A. Hoorfar, and N. Engheta, "Low profile antenna array considerations for through-the-wall microwave imaging (TWMI) applications," in Proc. IEEE AP-S Int. Symp., Washington, DC, 2005, pp. 338-341.

[15] F. Ahmad, M. G. Amin, and S. A. Kassam, "Synthetic aperture beamformer for imaging through a dielectric wall," IEEE Trans. Aerosp. Electron. Syst., vol. 41, no. 1, pp. 271-283, Jan. 2005.

[16] G. Wang, Y. Zhang, and M. Amin, "A new approach for target locations in the presence of wall ambiguities," IEEE Trans. Aerosp. Electron. Syst., vol. 42, no. 1, pp. 301-315, Jan. 2006.

[17] M. G. Amin, "Radar, signal, and image processing techniques for through the wall imaging," Proc. SPIE, vol. 5819, pp. 33-45, Jun. 2005.

[18] M. P. Rowe, E. N. Pugh, Jr., J. S. Tyo, and N. Engheta, "Polarizationdifference imaging: A biologically inspired technique for observation through scattering media," Opt. Lett., vol. 20, no. 6, pp. 608-610, Mar. 1995.

[19] J. S. Tyo, E. N. Pugh, Jr., and N. Engheta, "Colorimetric representation for use with polarization-difference imaging of objects in scattering media," J. Opt. Soc. Amer. A, Opt. Image Sci., vol. 15, no. 2, pp. 367-374, Feb. 1998

[20] K. M. Yemelyanov, S.-S. Lin, W. Q. Luis, E. N. Pugh, Jr., and N. Engheta, "Bio-inspired display of polarization information using certain visual cues," Proc. SPIE, vol. 5158, pp. 71-84, Dec. 2003.

[21] K. M. Yemelyanov, S.-S. Lin, E. N. Pugh, Jr., and N. Engheta, "Bioinspired, adaptive algorithms for two-channel polarization sensing under various polarization statistics with nonuniform distributions," Appl. Opt., vol. 45 , no. 22 , pp. 5504-5520, Aug. 2006

[22] R. Wehner, "Neurobiology of polarization vision," Trends Neurosci., vol. 12, no. 9, pp. 353-359, Sep. 1989.

[23] K. M. Yemelyanov, J. A. McVay, N. Engheta, and A. Hoorfar, "Adaptive polarization-difference imaging algorithms for through-the-wall microwave imaging scenarios," in Proc. IEEE AP-S Int. Symp., Washington, DC, 2005, pp. 114-117.

[24] K. S. Kunz and R. J. Luebbers, The Finite Difference Time Domain Method for Electromagnetics. New York: CRC Press, 1993.

[25] M. Born and E. Wolf, Principles of Optics. Cambridge, U.K.: Cambridge Univ. Press, 1969.

[26] I. T. Jolliffe, Principal Component Analysis. New York: SpringerVerlag, 1986.

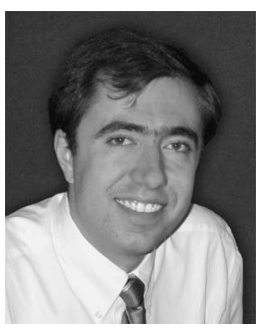

Konstantin M. Yemelyanov (S'99-M'01) received the M.S. (with honors) and Ph.D. degrees in electrical engineering from V. N. Karazin Kharkov National University, Kharkov, Ukraine, in 1996 and 2001, respectively.

He was a Postdoctoral Research Fellow with the University of Pennsylvania, Philadelphia. Since 2006, he has been a Research Assistant Professor with the Center for Advanced Communications, Department of Electrical and Computer Engineering, Villanova University, Villanova, PA. His research interests are in through-the-wall radar imaging, polarization imaging, and antenna design.

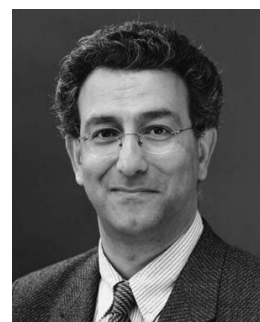

Nader Engheta (S'80-M'82-SM'89-F'96) received the B.S. degree in electrical engineering from the University of Tehran, Tehran, Iran, in 1978 and the M.S. and Ph.D. degrees in electrical engineering from the California Institute of Technology (Caltech), Pasadena, in 1979 and 1982, respectively.

After spending one year as a Postdoctoral Research Fellow with Caltech and four years as a Senior Research Scientist with the Dikewood Division, Kaman Sciences Corporation, Santa Monica, CA, in 1987, he joined the faculty of the University of Pennsylvania (UPenn), Philadelphia, where he is currently the H. Nedwill Ramsey Professor of Electrical and Systems Engineering with the Department of Electrical and Systems Engineering and a member of the David Mahoney Institute of Neurological Sciences and where he also holds an appointment with Department of Bioengineering. He was the Graduate Group Chair of electrical engineering from July 1993 to June 1997. His current research interests and activities span over a broad range of areas including metamaterials and plasmonics, nanooptics and nanophotonics, nanocircuit and nanostructure modeling, bio-inspired/biomimetic polarization imaging and reverse engineering of polarization vision, miniaturized antennas and nanoantennas, hyperspectral sensing, biologically based visualization and physics of sensing and display of polarization imagery, through-wall microwave imaging and sensing, millimeter-wave lensing systems, and fractional operators and fractional paradigm in electrodynamics. He has guest edited/coedited several special issues, namely, the special issue of the Journal of Electromagnetic Waves and Applications on the topic of "Wave Interaction with Chiral and Complex Media" in 1992, the part special issue of the Journal of the Franklin Institute on the topic of "Antennas and Microwaves (from the 13th Annual Benjamin Franklin Symposium) in 1995, the special issue of Wave Motion on the topic of "Electrodynamics in Complex Environments" (with L. B. Felsen) in 2001, and the special section of the journal Solid State Communications on the topic of "Negative Refraction and Metamaterials for Optical Science and Engineering" (with G. Shvets) in 2008. He coedited (with R. W. Ziolkowski) the book Metamaterials: Physics and Engineering Explorations (Wiley-IEEE Press, 2006). He was an Associate Editor of Radio Science (1991-1996) and was on the editorial board of the Journal of Electromagnetic Waves and Applications.

He has guest edited/coedited the special issue of the IEEE TRANSACTIONS ON ANTENNAS AND PROPAGATION on the topic of "Metamaterials" (with R. W. Ziolkowski) in 2003. He was an Associate Editor of the IEEE ANTENNAS AND Wireless Propagation LetTers (2002-2007) and the IEEE TRANSACTIONS ON ANTENNA AND PRopagation (1996-2001). He was an IEEE Antennas and Propagation Society Distinguished Lecturer for the period 1997-1999. He is a member of the American Association for the Advancement of Science, Sigma Xi, Commissions B, D, and K of the U.S. National Committee (USNC) of the International Union of Radio Science (URSI), and the Electromagnetics Academy. He was the Vice Chair/ChairElect of Commission B of USNC-URSI for 2006-2008 and is currently the Chair of this commission (2009-2011). He was the Chair (1989-1991) and Vice Chair (1988-1989) of the joint chapter of the IEEE Antennas and Propagation/Microwave Theory and Techniques in the Philadelphia Section. He served as a member of the Administrative Committee of the IEEE Society of Antennas and Propagation from January 2003 until December 2005. He has been elected to serve as the Vice Chair of the Gordon Research Conference on Plasmonics in 2010 and its Chair in 2012. He is a Guggenheim Fellow, a recipient of the IEEE Third Millennium Medal, and a Fellow of the Optical Society of America and the American Physical Society. He is the recipient of various awards and distinctions for his scholarly research contributions and teaching activities including his selection as one of the Scientific American magazine's 50 leaders in science and technology in 2006 for developing the concept of optical lumped nanocircuits, the 2008 George H. Heilmeier Award for Excellence in Research, the UPS Foundation Distinguished Educator Term Chair, the Fulbright Naples Chair award, a National Science Foundation Presidential Young Investigator award, the Christian F. and Mary R. Lindback Foundation Award, and the W. M. Keck Foundation's 1995 Engineering Teaching Excellence Award. He is a two-time recipient of the S. Reid Warren, Jr. Award from UPenn's School of Engineering and Applied Science. 


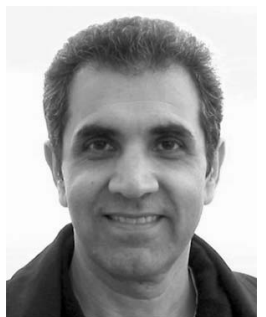

Ahmad Hoorfar (S'78-M'84-SM'95) received the B.S. degree in electrical engineering from the University of Tehran, Tehran, Iran, in 1975 and the M.S. and $\mathrm{Ph} . \mathrm{D}$. degrees in electrical engineering from the University of Colorado, Boulder, in 1978 and 1984, respectively.

He is a Professor of electrical and computer engineering, the Director of the Antenna Research Laboratory, Center for Advanced Communications, and the Program Director of the Department of Electrical and Computer Engineering's graduate admission and advising with Villanova University, Villanova, PA. Before joining Villanova University in 1988, he was a Postdoctoral Research Fellow with the Electromagnetics Laboratory, University of Colorado (1984-1986) and a Research Faculty with the National Science Foundation Research Center for Microwave/Millimeter-waves Computer-Aided Design, Boulder (1986-1988). He spent his sabbatical leave in 2002 with the NASA Jet Propulsion Laboratory, Pasadena, CA, where he contributed to the development of a general evolutionary optimization code for the design of feed horns for the NASA's deep space communication network. His present research interests include electromagnetic field theory, multifunction and reconfigurable antennas, miniaturized and fractal antennas, metamaterial media and surfaces, electromagnetic aspects of through-wall sensing and imaging, and evolutionary computational methods. $\mathrm{He}$ has published numerous journal articles and conference papers and has organized many special sessions at various international symposiums in these areas.

Dr. Hoorfar was the General Chair and Organizer of the 12th and 13th Benjamin Franklin Symposiums in Microwave and Antenna Technology held in 1994 and 1995 and the Co-organizer of the 22nd Antenna Measurement Technique Association Symposium in 2000. In 1995, he was the recipient of the Philadelphia section "IEEE chapter of the year award" for his leadership in chairing the Antennas and Propagation (AP)/Microwave Theory and Techniques joint chapter from 1993-1995. He has served as a Reviewer for various IEEE and other technical publications on antennas and microwaves in the last 20 years. He has also been on the technical program committees of numerous international symposia and conferences, including the IEEE AP Society Symposium, the IEEE Aerospace Conference, the IEEE Radio and Wireless Symposium, the International Union of Radio Science (URSI) Symposium, and the Progress in Electromagnetic Research Symposium. He is a member of URSI, Commission B. He was the recipient of Villanova University's Outstanding Faculty Research Scholar Award in 2007.

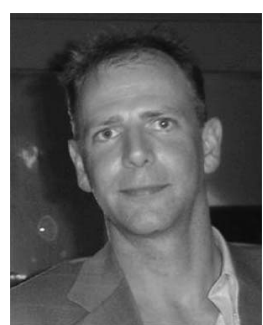

John A. McVay (S'01-M'07) received the B.S. degree (cum laude) in electrical engineering, with a minor in computer engineering, the M.S. degree in electrical engineering, and the Ph.D. degree in multidisciplinary engineering from Villanova University, Villanova, PA, in 2001, 2003, and 2006, respectively, where he was the first graduate from the multidisciplinary Ph.D. College of Engineering program.

He has been a Research Scientist with Eureka Aerospace, Pasadena, CA, since 2007, where he is currently working on projects including Impulse SAR through-the-wall Imaging System and High-Power Electromagnetic System for stopping vehicles and efficient small antennas. His research interests include metamaterials, with an emphasis on antenna applications, electrically small antennas, through-the-wall imaging, ultrawideband antennas, and scale model measurements of electrically large platforms (aircraft, ships, etc.). He has more than 50 technical publications including a chapter in the book Metamaterials: Physics and Engineering Explorations (Wiley-IEEE Press, 2006).

In 2006, he was the first recipient of Villanova University's Center for Advanced Communications Student of the Year Award. He was a finalist for the Student Paper Competition at the IEEE International Symposium on Antennas and Propagation in 2005 and was awarded an internship through the Office of Naval Research Enterprise Internship Program in 2004. He received first place in the 2003 IEEE Sarnoff Symposium Student Paper Competition. 